\title{
Prenatal exposure to antidepressants and persistent pulmonary hypertension of the newborn: systematic review and meta-analysis
}

\begin{abstract}
Objective To examine the risk for persistent pulmonary hypertension of the newborn associated with antenatal exposure to antidepressants.

Design Systematic review and meta-analysis.

Data sources Embase, Medline, PsycINFO, and CINAHL from inception to 30 December 2012.

Eligibility English language studies reporting persistent pulmonary hypertension of the newborn associated with exposure to antidepressants. Two independent reviewers extracted data and assessed the quality of each article.

Results Of the 3077 abstracts reviewed, 738 papers were retrieved and seven included. All seven studies were above our quality threshold. Quantitative analysis was only possible for selective serotonin reuptake inhibitors (SSRIs). Although exposure to SSRIs in early pregnancy was not associated with persistent pulmonary hypertension of the newborn (odds ratio 1.23 , 95\% confidence interval 0.58 to $2.60 ; P=0.58$ ), exposure in late pregnancy was $(2.50,1.32$ to $4.73 ; P=0.005)$. Effects were not significant for any of the moderator variables examined, including study design, congenital malformations, and meconium aspiration. It was not possible to assess for the effect of caesarean section, body mass index, or preterm delivery. The absolute risk difference for development of persistent pulmonary hypertension of the newborn after exposure to
\end{abstract}

SSRIs in late pregnancy was 2.9 to 3.5 per 1000 infants; therefore an estimated 286 to 351 women would need to be treated with an SSRI in late pregnancy to result in an average of one additional case of persistent pulmonary hypertension of the newborn.

Conclusions The risk of persistent pulmonary hypertension of the newborn seems to be increased for infants exposed to SSRIs in late pregnancy, independent of the potential moderator variables examined. A significant relation for exposure to SSRIs in early pregnancy was not evident. Although the statistical association was significant, clinically the absolute risk of persistent pulmonary hypertension of the newborn remained low even in the context of late exposure to SSRIs.

\section{Introduction}

Decisions about using antidepressants to treat major depressive disorder during pregnancy are complex and require the weighing of several variables. One potential adverse effect to be considered may be persistent pulmonary hypertension of the newborn. ${ }^{1-3}$ This condition is a relatively rare outcome, with an estimated baseline prevalence of 1.9 per 1000 live births. ${ }^{4}$ Normally the blood vessels in the lungs of the infants relax following delivery, but in persistent pulmonary hypertension of the newborn the resistance in the pulmonary vasculature following birth continues, leading to poor oxygenation. It is 
evident soon after birth, and symptoms can range in severity from mild respiratory distress to the most severe form, with hypoxia necessitating intensive medical care. ${ }^{5}$ Persistent pulmonary hypertension of the newborn has been defined as "a final common pathway of a variety of risk factors and insults that can cause pulmonary underdevelopment, maldevelopment, or poor postnatal adaptation." ${ }^{\prime 6}$ In 2006, Health Canada and the US Food and Drug Administration issued advisories ${ }^{78}$ alerting clinicians to a potential association between maternal use of selective serotonin reuptake inhibitors (SSRIs) during pregnancy and persistent pulmonary hypertension of the newborn following a publication showing such an association. With the accumulation of more research, the US advisory was revised in late 2011 to state that "given the conflicting results from different studies, it is premature to reach any conclusion about a possible link between SSRI use in pregnancy" and persistent pulmonary hypertension of the newborn. ${ }^{9}$ Indeed, in the scientific literature, evidence on the association between SSRI use in pregnancy and persistent pulmonary hypertension of the newborn is contradictory, ${ }^{6}$ with some studies finding no association, ${ }^{10-13}$ others finding some association, ${ }^{1-3}$ and yet others reporting differential findings depending on the timing of exposure (late versus early pregnancy). ${ }^{23}$ Recently, three reviews have been conducted on this topic: the first concluded that a small statistically increased risk exists, ${ }^{5}$ the second that exposure to SSRIs in late pregnancy should be considered, among others, as a potential risk for persistent pulmonary hypertension of the newborn, ${ }^{14}$ and the third that data supporting an association between persistent pulmonary hypertension of the newborn and exposure to SSRIs was "weak." To date no meta-analysis has been published to summarise the data and potentially help resolve these conflicting findings for clinicians.

Although persistent pulmonary hypertension of the newborn is not common, and causal mechanisms can be numerous, some risk factors are known (some more common than others). These risk factors include, but are not limited to, certain congenital malformations, premature birth, meconium aspiration, maternal obesity, and caesarean section mode of delivery. ${ }^{6}{ }^{15}$ However, many of the current studies on this topic examine exposure to SSRIs in isolation and do not exclude or control for these or other known risk factors. As several causative factors potentially exist, examining one association in isolation may be misleading as more than one risk factor may need to be present for the development of persistent pulmonary hypertension of the newborn. ${ }^{6} 14$

We performed a systematic review and meta-analysis to examine what, if any, relationship exists between any prenatal exposure to antidepressants and persistent pulmonary hypertension of the newborn (as defined by the authors in each study; see supplementary table $\mathrm{e}^{1-3}$ 10-13 $)$. We pooled data based on the timing of exposure (such as exposure to antidepressants in early versus late pregnancy), and where possible assessed for the effects of potential moderating variables, including original study quality and design, and the following known risk factors for persistent pulmonary hypertension of the newborn: preterm birth, congenital malformation, meconium aspiration, maternal obesity, and caesarean section mode of delivery.

\section{Methods}

\section{Search strategy and study selection}

This work is part of a large programme of research, and the methods have been previously published by our research team. ${ }^{16}$ In accordance with the Meta-analysis of Observational Studies in Epidemiology guidelines, ${ }^{17}$ for the larger programme of research two professional librarians with expertise within the areas of both psychopharmacology and psychiatry carried out independent literature searches up to 30 June 2010. Two further, updated independent searches were completed for persistent pulmonary hypertension of the newborn specifically to 30 December 2012. Keyword combinations used included: depressive/mood disorder, pregnancy/pregnancy trimesters, antidepressant drugs, infant outcome, and persistent pulmonary hypertension of the newborn (a full list of keywords is available from the authors on request). We utilised the following databases (from inception to 30 December 2012): Medline In-Process (Ovid), Medline (Ovid) to access current literature (searching for keywords only), PsycINFO (American Psychological Association; Ovid), Embase (Excerpta Medica, Elsevier; Ovid), CINAHL (Nursing; Allied Health), and Scopus (Elsevier) to access current literature (through searching for keywords only). We found no further articles through a search of the reference lists of reviews as well as meta-analyses.

\section{Inclusion and exclusion criteria}

Cohort and case-control English language studies that reported original data were eligible to be included if they reported on persistent pulmonary hypertension of the newborn after any exposure to antidepressants, had a comparison group of unexposed pregnant women for the antidepressant under analysis, and, if an effect size was not already provided, included enough data from which one could be calculated. We excluded abstracts, conference proceedings, and unpublished data as part of the search strategy methodology for the larger programme of research, ${ }^{16}$ owing to the number of studies potentially eligible and the lack of important details in their short text. Furthermore, if more than one paper had been published utilising the same cohort, we selected for inclusion the most recent study with the most number of cases of persistent pulmonary hypertension of the newborn.

\section{Data extraction}

Detailed data extraction and quality assessment methods have been described in a previous publication. ${ }^{16}$ Two research assistants independently screened the titles and abstracts of all studies and retrieved eligible papers. For each eligible study, data extraction forms were completed, modelled after the Strengthening the Reporting of Observational Studies in Epidemiology (STROBE) statement criteria. ${ }^{18}$ The forms included the source, study design, participants (sample, control, demographics, and clinical characteristics), inclusion and exclusion criteria, antidepressants examined, dosage, duration of exposure, primary and secondary outcomes, outcome assessment methods, and loss to follow-up. Where these were not provided in the original publication we sent requests for raw data to the authors of studies. We extracted adjusted estimates along with their variances if available, otherwise we used the published data to compute crude odds ratios and sample variances. When calculating the risk estimate, we added 0.5 to all cells with a 0 cell count. The primary outcome of interest for this meta-analysis was persistent pulmonary hypertension of the newborn, as defined by the authors of the original publications.

\section{Quality assessment}

Quality assessment was conducted for this programme of research utilising our previously published quality assessment tool (the Systematic Assessment of Quality in Observational Research (SAQOR). ${ }^{16}$ The SAQOR is based on quality 
assessment tools by Downs and Black ${ }^{19}$ and the Newcastle-Ottawa scale, ${ }^{20}$ and was developed specifically for this area of research. For each study, research assistants assessed 19 criteria within the following categories: sample, control group, quality of exposure or outcome measure, follow-up, and distorting influences. The last category included evaluation of whether studies controlled for confounders-for example, depression and other psychotropic drugs. We used a modification of the Grading of Recommendations Assessment, Development and Evaluation (GRADE) system ${ }^{21}$ and our quality assessment tool to assign a final rating of high, moderate, low, or very low to each article. Studies of high, moderate, or low quality were categorised as being above quality threshold, with studies of very low quality considered below quality threshold. The principal investigators resolved any differences in data extraction or quality assessment results between raters through consensus.

\section{Statistical analyses}

As persistent pulmonary hypertension of the newborn is a rare event we included adjusted or unadjusted odds ratios, prevalence, or relative risks in the analyses as estimates of odds ratios. If available, we preferred adjusted risk estimates, and we calculated odds ratios from raw numbers if not reported. Wilson et $\mathrm{al}^{12}$ reported an odds ratio of zero from a matched case-control study; to include this study, we needed a non-zero odds ratio. To calculate a non-zero odds ratio, we utilised values ranging from 0.0001 to 0.1 , and found the summary odds ratio varied by $<0.15$. We used an odds ratio of 0.001 for the non-zero odds ratio, as this gave a summary odds ratio that was the same with or without the study included. We used the Dersimonian and Laird random effects model ${ }^{22}$ to pool estimates of the odds ratios. For outcomes with only two published studies, we calculated the pooled estimate using the fixed effects model. To assess for publication bias in analyses with sufficient studies, we visually inspected a funnel plot of the study estimates (on the log scale for odds ratios) against their standard error, and we used Duval and Tweedie's trim and fill method ${ }^{23}$ to determine the number of missing studies, adjust for potential publication bias, and estimate adjusted exposure effects. We also conducted sensitivity analyses to determine the influence of any individual study by excluding each study one by one and recalculating the pooled effect. Study heterogeneity was evaluated with Cochrane's Q and quantified with $\mathrm{I}^{2}$, using a random effects model.

A small $\mathrm{I}^{2}$ and a non-significant value of the $\mathrm{Q}$ statistic suggest an underlying common effect, with the source of observed variation being largely random variation rather than heterogeneity. Commonly used benchmarks for $\mathrm{I}^{2}$ are that $25 \%$, $50 \%$, and $75 \%$ represent low, moderate, and high degrees of heterogeneity, respectively. ${ }^{24} \mathrm{We}$ used a priori specified subgroup analyses, carried out independently of the significance of Q, to explore sources of heterogeneity: study quality, study design (cohort or case-control), preterm birth, if congenital malformations or meconium aspiration were controlled for or excluded, maternal obesity, and whether the effect of caesarean section as a mode of delivery was assessed. Given the small sizes of the subgroups, these analyses used only fixed effects models to estimate effects within subgroups. Statistics for comparisons between subgroups are shown for both fixed and random effects models. Statistical analyses were conducted with the metafor package ${ }^{25}$ in $\mathrm{R}(3.0 .1),{ }^{26}$ and were similar to our other work..$^{27} 28$

\section{Results}

Of the 3077 abstracts reviewed, 2339 were excluded on the basis of the title and abstract. In total, 738 articles were retrieved and assessed for eligibility. Eight articles met the inclusion criteria, but one ${ }^{29}$ was excluded as it was based on the same cohort as a more recent study. ${ }^{1}$ Seven studies were included in the quantitative analysis (fig $1 \Downarrow,{ }^{30}$ see supplementary table ${ }^{1-3} 10-13$ ). Although four studies did report data for women using any antidepressant, ${ }^{1-3} 10$ not enough comparable data were available to permit pooling of at least three studies, and thus we limited our quantitative analysis to use of selective serotonin reuptake inhibitors (SSRIs, as in the individual study's definitions). Three studies reported data on exposure to SSRIs in early pregnancy, ${ }^{1-3}$ two on exposure at any time or any point in pregnancy, ${ }^{21}$ two on exposure for most or all of pregnancy, ${ }^{13}$ and five on exposure in late pregnancy. ${ }^{1-3} 1012$ Five studies used a cohort study design ${ }^{131011} 13$ and two a case-control study design. ${ }^{212}$ All of the studies were above our quality threshold, and as a result we did not perform subanalyses to examine the effect of study quality. Regarding preterm birth, two studies excluded infants aged $\leq 34$ gestational weeks, two studies excluded infants aged $<34$ weeks, one study excluded infants aged $\leq 33$ weeks, one study seemed to exclude infants aged $<36$ weeks, and one did not specify. In the late exposure analysis, where there were enough studies to permit the examination of moderator variables, four of five studies excluded infants in a similar manner for gestational age cut-offs (four excluded $<34$ or $\leq 34$ weeks, and one excluded $\leq 33$ weeks), thus "preterm birth" was not further assessed as a potential effect modifier. We did not assess caesarean section as a potential moderator variable, as only one study assessed the effect in combination with exposure to SSRIs and reported that this mode of delivery did not significantly modify the risk of persistent pulmonary hypertension of the newborn ${ }^{3}$; the authors did not provide an adjusted risk estimate. We were also unable to assess for maternal obesity as a potential moderating variable in our analyses. Of the three studies ${ }^{1-3}$ that did consider maternal body mass index as a potential confounding factor, two looked at prepregnancy body mass index and the other at early pregnancy body mass index; one of these studies ${ }^{3}$ dropped the variable after it showed no effect on the outcome in a subset analysis and so was not further adjusted for in their analyses. A fourth study ${ }^{12}$ considered a body mass index of more than 30 in an analysis of obesity but did not provide data for antidepressant users versus non-users. Overall, six of the seven studies accounted for confounders in their analyses (see supplementary table).

\section{Exposure to SSRIs}

Early pregnancy-For the three eligible studies ${ }^{1-3}$ with data on exposure to SSRIs in early pregnancy the pooled odds ratio was 1.23 (95\% confidence interval 0.58 to $2.60 ; \mathrm{P}=0.58$; fig $2 \Downarrow$ ). Significant heterogeneity was found across studies $\left(\mathrm{Q}_{2}=9.00\right.$, $\mathrm{P}=0.01$; table $1 \Downarrow)$, in the high range $\left(\mathrm{I}^{2}=77.8 \%\right)$.

Any time in pregnancy-The pooled odds ratio of the two eligible studies ${ }^{211}$ for exposure to SSRIs at any time and persistent pulmonary hypertension of the newborn was 1.55 (95\% confidence interval 0.79 to $3.04 ; \mathrm{P}=0.20$ ). No significant heterogeneity was found across studies $\left(\mathrm{Q}_{1}=0.14, \mathrm{P}=0.71\right.$, $\mathrm{I}^{2}=0.0 \%$; table 1 ).

Most or all of pregnancy-The pooled odds ratio of the two studies that classified exposure as "most or all of pregnancy"1 13 was significant $(3.33,1.58$ to $7.02 ; \mathrm{P}=0.002)$. No significant heterogeneity was found across the studies $\left(\mathrm{Q}_{1}=0.18, \mathrm{P}=0.67\right.$, $\mathrm{I}^{2}=0.0 \%$; table 1 ). 
Late pregnancy - The pooled odds ratio of the five studies that reported data on exposure to SSRIs in late pregnancy and persistent pulmonary hypertension of the newborn ${ }^{1-3} 10{ }^{12}$ was significant $(2.50,1.32$ to 4.73 ; $P=0.005$; fig $3 \Downarrow)$. Study heterogeneity was not significant, although the $\mathrm{I}^{2}$ was in the moderate degree range of heterogeneity $\left(\mathrm{Q}_{4}=8.31, \mathrm{P}=0.08\right.$, $\mathrm{I}^{2}=51.9 \%$; table 1$)$. The potential moderator variables of study design (case-control versus cohort), congenital malformations, and meconium aspiration (table 1) did not account for significant sources of heterogeneity.

\section{Publication bias and influential studies}

We assessed publication bias of the exposure in late pregnancy as this was the only grouping where data were sufficient to produce a funnel plot. Publication bias was indicated by the asymmetry of the funnel plot. The Duval and Tweedie trim and fill method imputed two missing studies, resulting in a slightly higher revised estimate of 2.84 (95\% confidence interval 1.41 to $5.72 ; \mathrm{P}=0.004)$.

The studies by Kieler et $\mathrm{al}^{3}$ and Reis and Källén ${ }^{1}$ accounted for most of the relative weight of the analysis for exposure in late pregnancy ( $41.5 \%$ and $29.8 \%$, respectively). In sensitivity analyses, we recalculated the pooled risk, excluding the studies one by one. With the exception of one study, ${ }^{1}$ the odds ratios remained significant when each study was excluded, including Kieler et $\mathrm{al}^{3}{ }^{3}$ with odds ratios between 2.11 and 2.81 . When we excluded the study by Reis and Källén ${ }^{1}$ the odds ratio remained within this range (odds ratio of 2.4) but was marginally non-significant $(\mathrm{P}=0.06)$.

\section{Estimates of absolute risk difference and number needed to treat to harm}

Given that persistent pulmonary hypertension of the newborn is rare, the odds ratio approximates the relative risk so we can multiply the baseline risk of $1.9 / 1000$ by the odds ratio to obtain the absolute risk for the exposure groups. Our pooled odds ratio of 2.5 for exposure in late pregnancy resulted in an absolute risk difference of 2.85/1000 live births and a number needed to treat to harm (NNTH) of 351 women. Accounting for two potentially missing studies, the revised odds ratio of 2.84 for exposure to SSRIs in late pregnancy led to an absolute risk difference of 3.50/1000 live births. The corresponding NNTH was 286 women. For exposure to SSRIs in early pregnancy, the odds ratio of 1.23 led to an absolute risk difference of $0.44 / 1000$ live births and an NNTH of 2288 women (table $2 \Downarrow$ ).

\section{Discussion}

The results of our meta-analysis suggest that the risk for persistent pulmonary hypertension of the newborn is increased with exposure to selective serotonin reuptake inhibitors (SSRIs) in late pregnancy; we did not find an increased risk from exposure in early pregnancy. (Although we aimed to examine exposure to all antidepressants, we limited our analyses to SSRIs, given the paucity of data on other antidepressant classes.) Our moderator analyses did not find study design, congenital malformations, or meconium aspiration to be significant effect modifiers. All the studies were above our quality threshold and we were not able to examine if caesarean section mode of delivery accounted for significant heterogeneity. We were also unable to examine maternal obesity as a possible moderating effect. In the late exposure analysis, the infants included were of either 33 or 34 weeks or more gestation, and thus we were unable to evaluate preterm birth as a source of heterogeneity as well. The analysis of exposure to SSRIs throughout gestation was also significant, whereas exposure at any time was not; however, both of these analyses were based on the pooling of only two studies and thus the data were insufficient to permit meaningful results and should be read with caution. We estimated risk differences to facilitate interpretation of our results for clinical practice (table 2). Although the pooled odds ratio of 2.5 for exposure to SSRIs in late pregnancy was statistically significant, it is important to consider that the low incidence of persistent pulmonary hypertension of the newborn in the general population means that the absolute increase resulting from such exposure would still be relatively low. We estimate that we would have to treat 286 to 351 women with an SSRI in late gestation to see one additional case of persistent pulmonary hypertension of the newborn. To our knowledge, ours is the first published meta-analysis to examine the association of exposure to SSRIs and persistent pulmonary hypertension of the newborn.

\section{Strengths and limitations of this study}

The strength of our work rests on the attention to potential confounding factors. We were only able to assess for the effects of potential modifying variables on the pooled effects in our group exposed to SSRIs in late pregnancy. Heterogeneity in the late exposure group was not significant (although the $\mathrm{I}^{2}$ was indicative of medium level heterogeneity) and, not surprisingly, none of the potential moderator variables accounted for significant heterogeneity. As the late exposure group was based on only five studies, the lack of significance for the moderator variable analysis may be a reflection of the limited number of studies that were pooled. Our modified pooled effect, following our assessment of publication bias using statistical estimates, resulted in a marginally higher odds ratio, suggesting that positive studies were missing.

Although meta-analytical techniques pool all available data, limitations include those of the original articles. Despite our attempt to determine if an association exists between all antidepressants with persistent pulmonary hypertension of the newborn, we were only able to examine SSRIs because of the lack of data on the other antidepressant classes; future research should determine if other classes of antidepressants show similar associations. The difference in definition of early versus late exposure among the studies was a further limitation. The early exposure analysis was not significant whereas the later one was significant. The early grouping included women exposed from three months before the start of pregnancy until 55 days' gestation ( $<8$ weeks), ${ }^{3}$ before week 20 of pregnancy, ${ }^{2}$ and before the first antenatal visit. ${ }^{1}$ The late exposure included women exposed after week 20 of pregnancy, ${ }^{2}{ }^{12}$ in week 20 or later (from 140 days' gestation until birth), ${ }^{3}$ in the third trimester, ${ }^{10}$ or after the first antenatal visit. ${ }^{1}$ Owing to the small number of studies eligible for inclusion in these analyses, it was not possible to assess further for this potential confounder beyond a sensitivity analysis for the late exposure subgroup. Removing the study ${ }^{1}$ that included an earlier definition of late exposure (after the first antenatal visit which is generally after the first trimester although unknown when exactly) resulted in a marginally non-significant result. Interestingly, both the early and the late exposure groups in the original study were significantly associated with persistent pulmonary hypertension of the newborn. A more uniform definition of early and late exposure in future research would be beneficial towards eliminating this potential confounding.

Our main analysis was based on five studies..$^{1-3} 1012$ Two of the studies ${ }^{13}$ potentially had some overlap in the study samples, as both included women from the Swedish medical birth register, with some overlap of the period sampled. These two studies did 
account for the most weight; however, the results were only altered when one of the studies ${ }^{1}$ was removed in sensitivity analyses $(\mathrm{P}=0.06)$. Another study ${ }^{10}$ had few cases of persistent pulmonary hypertension of the newborn and yet another study ${ }^{12}$ was underpowered. Although most of the studies did use adjusted data in an attempt to control for confounding influences, not all controlled for the same potential confounding variables (see supplementary table). Two studies, ${ }^{212}$ for example, did exclude congenital malformations known to be associated with persistent pulmonary hypertension of the newborn, but they did not exclude all malformations. Another study ${ }^{3}$ statistically controlled for several variables and excluded meconium aspiration in subanalyses, but did not exclude other known causes, leaving potential confounding. Moreover, we included this study ${ }^{3}$ in our analysis that controlled for meconium, as the authors reported "after excluding infants with meconium aspiration, the risk estimates increased slightly," and in the discussion they concluded that exclusion of meconium aspiration resulted in no major difference in risk but that the odds ratio with the exclusion in the group exposed to SSRIs in late pregnancy was not provided; thus in our "controlled for" analysis we used the odds ratio for infants with meconium aspiration. Three of the 33 infants with persistent pulmonary hypertension of the newborn in this study who were exposed to SSRIs in late pregnancy had meconium aspiration, one of the known risk factors for persistent pulmonary hypertension of the newborn. ${ }^{6}$ The lack of significance within our moderator analyses may be secondary to not only the small number of studies for inclusion but also the way in which the variables were handled in the studies pooled.

Although we wanted to assess for the effects of preterm birth, four of the five studies did not assess for gestational age but rather excluded infants born before at least 33 weeks. One study ${ }^{2}$ of the five did conduct an analysis restricted to full term births ( $\geq 37$ weeks) and found results similar to those when they had restricted births to greater than 34 weeks' gestation. However, persistent pulmonary hypertension of the newborn has been reported to be more common in infants born at 34 weeks compared with those born at term ( 37 to 41 weeks). ${ }^{29}{ }^{31}$ It is also important to note that preterm birth has been associated with both antidepressant use and maternal depression. ${ }^{28}{ }^{32}$ The independent effects of preterm birth and exposure to SSRIs remain to be determined.

Only one study in the late exposure analysis ${ }^{3}$ examined for the effect of caesarean section on the potential relation between prenatal exposure to SSRIs and persistent pulmonary hypertension of the newborn; however, it did not present an odds ratio with this mode of delivery controlled for or excluded. Rather, caesarean section was examined as an effect modifier and was determined to be non-significant $(\mathrm{P}>0.18)$. One study ${ }^{12}$ had no events of persistent pulmonary hypertension of the newborn in their group exposed to SSRIs and also examined caesarean section. That study ${ }^{12}$ found caesarean delivery before onset of labour to be associated with persistent pulmonary hypertension of the newborn (odds ratios $4.9,95 \%$ confidence interval 1.7 to 14.0 ). Whereas one study ${ }^{10}$ reported no increased risk of persistent pulmonary hypertension of the newborn after exposure to SSRIs in late pregnancy, the rates of caesarean section were "similar" between exposed and unexposed groups. The caesarean section mode of delivery, however, has been found to be more common in infants with persistent pulmonary hypertension of the newborn, ${ }^{33}{ }^{34}$ been considered a risk factor for persistent pulmonary hypertension of the newborn, ${ }^{6}$ and been noted in women with depression. ${ }^{35}$ It is possible that the increased prevalence for this mode of delivery may be due to maternal preference. ${ }^{36}$ The effect of maternal obesity was also not examined, as in the original papers the authors either did not examine it as a moderator or they analysed it in a way that was not comparable. As obesity is a known risk factor for persistent pulmonary hypertension of the newborn, ${ }^{33}$ and depression itself seems to be a reciprocal risk factor for obesity, ${ }^{38}$ the relation with persistent pulmonary hypertension of the newborn may be confounded. Future studies should control for caesarean section and consider obesity when exploring potential associations with persistent pulmonary hypertension of the newborn.

None of the five studies evaluated for the effects of a clinical depression on persistent pulmonary hypertension of the newborn. One study ${ }^{3}$ did show that women who were not using antidepressants while pregnant but who had a history of admission to hospital for a psychiatric condition also had an increased risk of delivering an infant with persistent pulmonary hypertension of the newborn (odds ratios 1.3, 95\% confidence interval 1.1 to 1.6). The adjusted odds ratio in those women who used SSRIs in late pregnancy and had a history of admission to hospital for a psychiatric condition was even higher at 3.1 (95\% confidence interval 1.9 to 4.9$)$, suggesting that the combination of a psychiatric disorder and antidepressant use might confer a higher risk. One study ${ }^{2}$ did note that maternal depression may not be independently associated with persistent pulmonary hypertension of the newborn, as the study did not find an association with the condition for non-SSRI antidepressants or when exposure to SSRIs was restricted to the first half of pregnancy. Although the effect of depression may not be direct, it is possible that in combination with other factors the risk for persistent pulmonary hypertension of the newborn after exposure to maternal depression may be attenuated-that is, depression in combination with caesarean section. Women with depression have been found to have an increase in some of the known risk factors for persistent pulmonary hypertension of the newborn, such as caesarean section, obesity, and preterm delivery, which may also confound the results and association. As has been often noted in research evaluating possible adverse outcomes after prenatal exposure to antidepressants, it is important to emphasise that antidepressants are also prescribed for other psychiatric conditions that may exert an unknown independent effect. SSRIs are prescribed for anxiety disorders, which are increasingly recognised as being common in pregnancy ${ }^{39}$ and are themselves associated with adverse birth outcomes. ${ }^{40-42}$

Another important limitation of the literature, and thus our meta-analysis, is that the included studies ${ }^{1-3}{ }^{10-13}$ either did or did not consistently control for or examine the severity of the persistent pulmonary hypertension of the newborn cases included, as well as the underlying factors that may result in higher mortality rates of these infants. Only three studies ${ }^{2} 310$ reported on infant outcome: one reported frequency of infant death within six months after delivery ${ }^{2}$; one reported on infant death, without specifying a time frame ${ }^{3}$; and one reported the mechanism of treatment for persistent pulmonary hypertension of the newborn and outcome (all resolved) ${ }^{10}$ (see supplementary table). Furthermore, some studies excluded cases with more or less severe complications (for example, congenital malformations, meconium aspiration, preterm delivery $(\leq 34$ weeks), neonatal pneumonia, or sepsis, see supplementary table), whereas others did not. This inconsistency thus inhibits further analysis of the severity of persistent pulmonary hypertension of the newborn within the context of SSRI exposure as a risk factor. 
Lastly, an association between exposure to SSRIs and persistent pulmonary hypertension of the newborn does not imply causation, as discussed by one study, ${ }^{15}$ particularly when there are questions around whether exposure has occurred (for example, in prescription based registry studies). The pooled effects were dominated by the registry studies, and yet we cannot be confident that the mothers used the drugs as prescribed. Data collected from prescription registries do not always precisely reflect that the drug was taken as prescribed. ${ }^{43}$ Moreover, there is also uncertainty regarding dose, length of use, and the method used for handling women who cease to use the medication at any point in pregnancy. ${ }^{44}$ In addition, when data are retrospective, potential bias can exist. In the case of one study, ${ }^{2}$ for example, the authors determined exposure retrospectively by telephone interview, and thus recall bias and consequently inflated or inaccurate odds ratios cannot be ruled out. Proof of exposure is fundamental for the meaningfulness of these study's findings, and yet it is not without question in the data associating exposure to SSRIs and persistent pulmonary hypertension of the newborn. ${ }^{15} 4344$

\section{Conclusions and policy implications}

Depression during pregnancy must not be left untreated, as the potential for untoward effects is not negligible and can extend into the postpartum period. Selection of treatment is based on several factors, and antidepressant drugs may be necessary, especially in severe depressive episodes. ${ }^{45}$ Although the odds for persistent pulmonary hypertension of the newborn seem to be greater with the use of SSRIs later in pregnancy, despite the limitations of the original studies, the risk is still low. Results from this meta-analysis still concur with earlier statements, ${ }^{3} 1546$ that fewer than about 1 infant in 100 will develop persistent pulmonary hypertension of the newborn after antenatal exposure to SSRIs. Although this condition is serious and death rates between $5 \%$ and $10 \%$ have been reported, when it is associated with other conditions (such as some congenital malformations, meconium aspiration, sepsis, and idiopathic disease), it can be managed favourably. ${ }^{46}$ The death rate in infants with persistent pulmonary hypertension of the newborn who have been exposed to SSRIs, however, remains unknown (although one study did report $9.1 \%$ of the infants died who were exposed to SSRIs compared with $9.5 \%$ of those who were not exposed ${ }^{3}$ ).

Conversely, although suicide during the perinatal period has not been well researched, there is some existing data, and during the postpartum period suicide may not be less common than suicide at other times of the life cycle in women. ${ }^{47}$ One study reported suicide to be the leading cause of maternal mortality in the United Kingdom, being responsible for $28 \%$ of maternal deaths ${ }^{47} ; 68 \%$ of the women who died by suicide had a major mental illness such as severe depression or psychosis. Others have also found maternal psychiatric illness to be a leading cause of maternal mortality. ${ }^{48}$ Although also poorly researched, a high proportion of infanticides (murder in the first year of life) have been cited as occurring in the context of postpartum psychiatric illness. ${ }^{49}$ Maternal psychiatric illness is also known to increase the risk of filicide (child murder by mother), ${ }^{50}$ and maternal filicide with suicide, although rare, has been found to occur in the setting of psychiatric illness (more than the majority of the mothers in one study were found to have depression ${ }^{51}$ ). It is imperative that the mother's health be weighed heavily in treatment decisions; she and her family must be counselled on both the risks of exposing the fetus to antidepressant drugs and the risks of severe depressive illness. Although more research controlling for risk factors discussed in this study as well as others not reviewed here, is needed, pregnant women considering or using SSRIs and their families should be educated about persistent pulmonary hypertension of the newborn, how the symptoms can range in severity, what treatments are available for it at the institution where the birth will take place, and that it can typically be managed successfully if it does occur in the context of SSRI use. To avoid delays in diagnosis and treatment of persistent pulmonary hypertension of the newborn, neonatologists must be made aware of exposure to SSRIs.

We thank the advisory committee members for valuable input and their expertise to this project: Hiltrud Dawson, Michael Dunn, Adrienne Einarson, Alicja Fishell, Jasmine Gandhi, Jan Kasperski, Sidney Kennedy, Diane Meschino, Irena Nulman, Sagar Parikh, Paula Ravitz, Sarah Romans, Simone Vigod, Jennifer Blake, and Karen Wade; and Lindsay Witton, Maura O'Keefe, and Svetlana Emelianova for providing their feedback during many research meetings. Sheila Lacroix and Ani Orchanian-Cheff devised search strategies and completed literature searches. Alex Kiss performed the preliminary meta-analyses. Jenna McKay, Allison Eady, and Kimberly Radford assisted with data extraction and quality assessment processes.

Contributors: SG and LER conceptualised and designed the study. All authors analysed and interpreted the data, drafted the article, or revised it critically for important intellectual content, and gave final approval of the version to be published. SG and LER are the guarantors. All authors, external and internal, had full access to all of the data in the study and can take responsibility for the integrity of the data and the accuracy of the data analysis.

Funding: This study was funded by a research syntheses grant from the Canadian Institutes of Health Research (KRS-83127) and the Ontario Ministry of Health and Long-Term Care through the Drug Innovation Fund (grant No 2008-005). SG held a new investigator award in women's health research from Canadian Institutes of Health Research in partnership with the Ontario Women's Health Council (award NOW-88207). LER held a new investigator award from the Canadian Institutes of Health Research and the Ontario Women's Health Council (award NOW-84656). The funding sources had no role in the study design; collection, analysis, or interpretation of results; writing of the manuscript; or submission of the manuscript for publication. The researchers were independent from the funders. The views expressed here do not necessarily reflect those of the Ministry of Health and Long-Term Care.

Competing interests: All authors have completed the ICMJE uniform disclosure form at www.icmje.org/coi_disclosure.pdf and declare: no support from any organisation for the submitted work [other than the granting agencies as above]; in the past three years, SG has received research grant support from the Canadian Institutes of Health Research and the Ontario Ministry of Health for the conduct of this study, and has received honorariums as a consultant, member of an advisory committee, or for lectures from Servier and Lundbeck, and research grant support from the CR Younger Foundation, outside the submitted work; LER has received research grant support from the Canadian Institutes of Health Research and the Ontario Ministry of Health and Long-Term Care for the conduct of this study; and MS has received grants from the Canadian Institutes of Health Research, Ontario Mental Health Foundation, Natural Sciences and Engineering Research Council of Canada, grants and personal fees from Society for Women's Health Research, grants from Pfizer and Eli Lilly, grants and personal fees from Lundbeck, personal fees from AstraZeneca, Bayer Canada, and Servier, all outside of the submitted work]; no other relationships or activities that could appear to have influenced the submitted work.

Ethical approval: Not required.

Data sharing: The dataset is available from the corresponding author at Sophie.Grigoriadis@sunnybrook.ca.

Transparency: The lead author (the manuscript's guarantor) affirms that this manuscript is an honest, accurate, and transparent account of the 


\section{What is already known on this topic}

Treatment decisions about the use of antidepressant drugs during pregnancy are multifactorial and must consider potential risks to the fetus/neonate

Contradictory evidence exists on the association between SSRI use during pregnancy and the development of persistent pulmonary hypertension of the newborn in the neonate

\section{What this study adds}

The risk for persistent pulmonary hypertension of the newborn in infants exposed to SSRIs during late pregnancy is small although significantly increased

The absolute risk of persistent pulmonary hypertension of the newborn remains low even in the context of exposure to SSRIs in late pregnancy

Given that persistent pulmonary hypertension of the newborn occurs in about 1.9/1000 livebirths, about 286 to 351 women would need to be treated with an SSRI during late gestation to result in an average of one associated case of persistent pulmonary hypertension of the newborn

study being reported; that no important aspects of the study have been omitted; and that any discrepancies from the study as planned (and, if relevant, registered) have been explained.

1 Reis M, Källén B. Delivery outcome after maternal use of antidepressant drugs in pregnancy: an update using Swedish data. Psychol Med 2010;40:1723-33.

Chambers CD, Hernandez-Diaz S, Van Marter LJ, Werler MM, Louik C, Jones KL, et al. Selective serotonin-reuptake inhibitors and risk of persistent pulmonary hypertension of the newborn. N Engl J Med 2006;354:579-87.

3 Kieler H, Artama M, Engeland A, Ericsson Ö, Furu K, Gissler M, et al. Selective serotonin reuptake inhibitors during pregnancy and risk of persistent pulmonary hypertension in the newborn: population based cohort study from the five Nordic countries. BMJ 2012;344:d8012

4 Walsh-Sukys MC, Tyson JE, Wright LL, Bauer CR, Korones SB, Stevenson DK, et al. Persistent pulmonary hypertension of the newborn in the era before nitric oxide: practice variation and outcomes. Pediatrics 2000;105:14-20.

5 't Jong GW, Einarson T, Koren G, Einarson A. Antidepressant use in pregnancy and persistent pulmonary hypertension of the newborn (PPHN): a systematic review. Reprod Toxicol 2012;34:293-7.

6 Occhiogrosso M, Omran SS, Altemus M. Persistent pulmonary hypertension of the newborn and selective serotonin reuptake inhibitors: lessons from clinical and translational studies. Am J Psychiatry 2012;169:134-40.

7 Archive-Newer antidepressants linked to serious lung disorder in newborns. 2013. www. healthycanadians.gc.ca/recall-alert-rappel-avis/hc-sc/2006/13080a-eng.php

8 US Food and Drug Administration. Public Health Advisory: treatment challenges of depression in pregnancy and the possibility of persistent pulmonary hypertension in newborns. 2012. www.fda.gov/Drugs/DrugSafety/

PostmarketDrugSafetyInformationforPatientsandProviders/ DrugSafetyInformationforHeathcareProfessionals/PublicHealthAdvisories/ucm 124348. htm

9 US Food and Drug Administration.: FDA Drug and Safety Communication: selective serotonin reuptake inhibitor (SSRI) antidepressant use during pregnancy and reports of a rare heart and lung condition in newborn babies. 2012. www.fda.gov/Drugs/DrugSafety/ ucm283375.htm

10 Andrade SE, McPhillips H, Loren D, Raebel MA, Lane K, Livingston J, et al. Antidepressant medication use and risk of persistent pulmonary hypertension of the newborn. Pharmacoepidemiol Drug Saf 2009;18:246-52.

11 Wichman CL, Moore KM, Lang TR, St Sauver JL, Heise RH Jr, Watson WJ. Congenita heart disease associated with selective serotonin reuptake inhibitor use during pregnancy. Mayo Clin Proc 2009;84:23-7.

12 Wilson KL, Zelig CM, Harvey JP, Cunningham BS, Dolinsky BM, Napolitano PG. Persistent pulmonary hypertension of the newborn is associated with mode of delivery and not with maternal use of selective serotonin reuptake inhibitors. Am J Perinatol 2011;28:19-24.

13 Lim K, Sanders A, Brain U, Riggs W, Oberlander TF, Rurak D. Third trimester fetal pulmonary artery Doppler blood flow velocity characteristics following prenatal selective serotonin reuptake inhibitor (SSRI) exposure. Early Hum Dev 2012;88:609-15.

14 Galbally M, Gentile S, Lewis AJ. Further findings linking SSRIs during pregnancy and persistent pulmonary hypertension of the newborn: clinical implications. CNS Drugs 2012;26:813-22.

15 Koren G, Nordeng H. SSRIs and persistent pulmonary hypertension of the newborn. BMJ 2012;344:d7642.

16 Ross LE, Grigoriadis S, Mamisashvili L, Koren G, Steiner M, Dennis CL, et al. Quality assessment of observational studies in psychiatry: an example from perinatal psychiatric research. Int J Methods Psychiatr Res 2011;20:224-34.

17 Stroup DF, Berlin JA, Morton SC, Olkin I, Williamson GD, Rennie D, et al. Meta-analysis of observational studies in epidemiology: a proposal for reporting: Meta-analysis of Observational Studies in Epidemiology (MOOSE) group. JAMA 2000;283:2008-12.

18 Von Elm E, Altman DG, Egger M, Pocock SJ, Gotzsche PC, Vandenbroucke JP, et al. The Strengthening the Reporting of Observational Studies in Epidemiology (STROBE) statement: guidelines for reporting observational studies. Lancet 2007;370:1453-7.

19 Downs SH, Black N. The feasibility of creating a checklist for the assessment of the methodological quality both of randomised and non-randomised studies of health care interventions. J Epidemiol Community Health 1998:52:377-84.

20 Wells GA, Shea B, O'Connell D, Petersen J, Welch V, Losos M, et al. The Newcastle-Ottawa Scale (NOS) for assessing the quality of nonrandomized studies in meta-analyses. 2009. www.ohri.ca/programs/clinical_epidemiology/oxford.htm

21 Guyatt GH, Oxman AD, Vist GE, Kunz R, Falck-Ytter Y, Alonso-Coello P, et al. GRADE: an emerging consensus on rating quality of evidence and strength of recommendations. BMJ 2008;336:924-6.

22 DerSimonian R, Laird N. Meta-analysis in clinical trials. Control Clin Trials 1986;7:177-88.

23 Duval S, Tweedie R. Trim and fill: a simple funnel-plot-based method of testing and adjusting for publication bias in meta-analysis. Biometrics 2000;56:455-63.
24 Huedo-Medina TB, Sanchez-Meca J, Marin-Martinez F, Botella J. Assessing heterogeneity in meta-analysis: Q statistic or I2 index? Psychol Methods 2006;11:193-206.

25 Viechtbauer W. Conducting meta-analyses in R with the metafor package. J Stat Softw 2010;36:1-48. 2012. www.jstatsoft.org/v36/i03/

26 The R Project for Statistical Computing. R: A language and environment for statistical computing. R Foundation for Statistical Computing. 2012. www.R-project.org/

27 Grigoriadis S, VonderPorten EH, Mamisashvili L, Eady A, Tomlinson G, Dennis CL, et al. The effect of prenatal antidepressant exposure on neonatal adaptation: a systematic review and meta-analysis. J Clin Psychiatry 2013;74:e309-20.

28 Grigoriadis S, VonderPorten EH, Mamisashvili L, Tomlinson G, Dennis CL, Koren G, et al. The impact of maternal depression during pregnancy on perinatal outcomes: a systematic review and meta-analysis. J Clin Psychiatry 2013;74:e321-41.

29 Källén BA, Olausson PO. Maternal use of selective serotonin reuptake inhibitors and risk of persistent pulmonary hypertension of the newborn. Pharmacoepidemiol Drug Saf 2008;17:801-6.

30 Moher D, Liberati A, Tetzlaff J, Altman DG, PRISMA Group. Reprint—preferred reporting items for systematic reviews and meta-analyses: the PRISMA statement. Phys Ther 2009;89:873-80

31 Hibbard JU, Wilkins I, Sun L, Gregory K, Haberman S, Hoffman M, et al. Respiratory morbidity in late preterm births. JAMA 2010;304:419-25.

32 Ross LE, Grigoriadis S, Mamisashvili L, VonderPorten EH, Roerecke M, Rehm J, et al. Selected pregnancy and delivery outcomes after exposure to antidepressant medication: a systematic review and meta-analysis. JAMA Psychiatry 2013;70:436-43.

33 Hernández-Díaz S, Van Marter LJ, Werler MM, Louik C, Mitchell AA. Risk factors for persistent pulmonary hypertension of the newborn. Pediatrics 2007;120:e272-82.

34 Winovitch KC, Padilla L, Ghamsary M, Lagrew DC, Wing DA. Persistent pulmonary hypertension of the newborn following elective cesarean delivery at term. J Matern Fetal Neonatal Med 2011;24:1398-402.

35 Chung TKH, Lau TK, Yip ASK, Chiu HFK, Lee DTS. Antepartum depressive symptomatology is associated with adverse obstetric and neonatal outcomes. Psychosom Med 2001;63:830-4

36 Hildingsson I, Rådestad I, Rubertsson C, Waldenström U. Few women wish to be delivered by caesarean section. BJOG 2002;109:618-23.

37 Wiklund I, Edman G. Andolf E. Cesarean section on maternal request: reasons for the request, self-estimated health, expectations, experience of birth and signs of depression among first-time mothers. Acta Obstet Gynecol Scand 2007;86:451-6.

38 Luppino FS, de Wit LM, Bouvy PF, Stijnen T, Cuijpers P, Penninx BW, Zitman FG. Overweight, obesity, and depression: a systematic review and meta-analysis of longitudinal studies. Arch Gen Psychiatry 2010;67:220-9.

39 Grigoriadis S, de Camps Meschino D, Barrons E, Bradley L, Eady A, Fishell A, et al. Mood and anxiety disorders in a sample of Canadian perinatal women referred for psychiatric care. Arch Womens Ment Health 2011;14:325-33.

40 Hernandez-Martinez C, Val VA, Murphy M, Busquets PC, Sans JC. Relation between positive and negative maternal emotional states and obstetrical outcomes. Women Health 2011;:51:124-35

41 De Paz NC, Sanchez SE, Huaman LE, Chang GD, Pacora PN, Garcia PJ, et al. Risk of placental abruption in relation to maternal depressive, anxiety and stress symptoms. $J$ Affect Disord 2011;130:280-4.

42 Groome LJ, Swiber MJ, Bentz LS, Holland SB, Atterbury JL. Maternal anxiety during pregnancy: effect on fetal behavior at 38 to 40 weeks of gestation. J Dev Behav Pediatr 1995;16:391-6.

43 Källén B, Nilsson E, Olausson PO. Antidepressant use during pregnancy: comparison of data obtained from a prescription register and from antenatal care records. Eur J Clin Pharmacol 2011;67:839-45.

44 Grzeskowiak LE, Gilbert AL, Morrison JL. Exposed or not exposed? Exploring exposure classification in studies using administrative data to investigate outcomes following medication use during pregnancy. Eur J Clin Pharmacol 2012;68:459-67.

45 Yonkers KA, Wisner KL, Stewart DE, Oberlander TF, Dell DL, Stotland N, et al. The management of depression during pregnancy: a report from the American Psychiatric Association and the American College of Obstetricians and Gynecologists. Gen Hosp Psychiatry 2009;31:403-13.

46 Koren G, Nordeng H. Antidepressant use during pregnancy: the benefit-risk ratio. Am J Obstet Gynecol 2012;207:157-63.

47 Oates M. Perinatal psychiatric disorders: a leading cause of maternal morbidity and mortality. Br Med Bull 2003;67:219-29.

48 Austin MP, Kildea S, Sullivan E. Maternal mortality and psychiatric morbidity in the perinatal period: challenges and opportunities for prevention in the Australian setting. Med J Aust 2007;186:364-7.

49 Spinelli M. Maternal infanticide associated with mental illness: prevention and the promise of saved lives. Am J Psychiatry 2004:161:1548-57.

50 Friedman SH, Resnick PJ. Child murder by mothers: patterns and prevention. World Psychiatry 2007;6:137-41. 
51 Friedman SH, Hrouda DR, Holden CE, Noffsinger SG, Resnick PJ. Filicide-suicide: common factors in parents who kill their children and themselves. J Am Acad Psychiatry Law 2005;33:496-504.

Accepted: 11 November 2013
Cite this as: BMJ 2014;348:f6932

This is an Open Access article distributed in accordance with the Creative Commons Attribution Non Commercial (CC BY-NC 3.0) license, which permits others to distribute, remix, adapt, build upon this work non-commercially, and license their derivative works on different terms, provided the original work is properly cited and the use is non-commercial. See: http://creativecommons.org/licenses/by-nc/3.0/. 


\section{Tables}

Table 1 | Exposure to selective serotonin reuptake inhibitors (SSRIs) and risk of persistent pulmonary hypertension of the newborn: results of meta-analyses

\begin{tabular}{|c|c|c|c|c|c|c|c|c|c|}
\hline \multirow[t]{3}{*}{ Analysis } & \multirow{3}{*}{$\begin{array}{l}\text { No of } \\
\text { studies }\end{array}$} & \multicolumn{5}{|c|}{ Within group } & \multicolumn{3}{|c|}{ Effect of moderator } \\
\hline & & \multirow[t]{2}{*}{ Odds ratio $(95 \% \mathrm{Cl})$} & \multirow[t]{2}{*}{$P$ value } & \multicolumn{3}{|c|}{ Heterogeneity } & \multirow[b]{2}{*}{ Q between studies } & \multirow[b]{2}{*}{$P$ value } & \multirow[b]{2}{*}{$\begin{array}{c}\mathrm{I}^{2} \text { (\% variance } \\
\text { explained) }\end{array}$} \\
\hline & & & & Q within studies & $P$ value & $I^{2}$ & & & \\
\hline \multicolumn{10}{|c|}{ Timing of exposure to SSRIs } \\
\hline Early pregnancy & 3 & $1.23(0.58 \text { to } 2.60)^{\star}$ & 0.58 & $9.00(\mathrm{df}=2)$ & 0.01 & 77.8 & & & \\
\hline Any time & $2 \dagger$ & 1.55 (0.79 to 3.04$) \ddagger$ & 0.20 & $0.14(\mathrm{df}=1)$ & 0.71 & 0.0 & & & \\
\hline Most or all of pregnancy & $2 \dagger$ & 3.33 (1.58 to 7.02$) \ddagger$ & 0.002 & $0.18(\mathrm{df}=1)$ & 0.67 & 0.0 & & & \\
\hline Late pregnancy: & 5 & $2.50(1.32 \text { to } 4.73)^{*}$ & 0.005 & $8.31(\mathrm{df}=4)$ & 0.08 & 51.9 & & & \\
\hline Study design: & & & & & & & $\begin{array}{c}2.85(\mathrm{df}=1) \ddagger, 0.31 \\
(\mathrm{df}=1)^{\star}\end{array}$ & $0.09 \neq, 0.58^{*}$ & $34.0 \ddagger, 4.0^{*}$ \\
\hline Case-control & 2 & 5.31 (1.94 to 14.56$) \ddagger$ & 0.001 & $4.48(\mathrm{df}=1)$ & 0.03 & 77.7 & & & \\
\hline Cohort & 3 & 2.14 (1.57 to 2.92$) \ddagger$ & $<0.001$ & $0.98(\mathrm{df}=2)$ & 0.61 & 0.0 & & & \\
\hline Congenital malformations: & & & & & & & $\begin{array}{c}2.85(\mathrm{df}=1) \ddagger, 0.31 \\
(\mathrm{df}=1)_{1}^{*}\end{array}$ & $0.09 \ddagger, 0.58^{*}$ & $34.0 \ddagger, 4.0^{*}$ \\
\hline Excluded & 2 & $5.31(1.94$ to 14.56$) \ddagger$ & 0.001 & $4.48(\mathrm{df}=1)$ & 0.03 & 77.7 & & & \\
\hline Uncontrolled & 3 & 2.14 (1.57 to 2.92$) \ddagger$ & $<0.001$ & $0.98(\mathrm{df}=2)$ & 0.61 & 0.0 & & & \\
\hline Meconium aspiration: & & & & & & & $\begin{array}{c}1.47(\mathrm{df}=1) \ddagger, 0.72 \\
(\mathrm{df}=1)^{*}\end{array}$ & $0.23 \ddagger, 0.40^{\star}$ & $18.0 \ddagger, 9.0^{*}$ \\
\hline Controlled for & 2 & 2.07 (1.46 to 2.93$) \ddagger$ & $<0.001$ & $3.50(\mathrm{df}=1)$ & 0.06 & 71.4 & & & \\
\hline Uncontrolled & 3 & $3.12(1.77$ to 5.48$) \ddagger$ & $<0.001$ & $3.35(\mathrm{df}=2)$ & 0.19 & 40.2 & & & \\
\hline
\end{tabular}

*Pooled effect size estimated using random effects model.

†Insufficient number of studies for meaningful meta-analyses.

$\ddagger$ Pooled effect size estimated using fixed effects model. 
Table 2| Risk of persistent pulmonary hypertension of the newborn and number needed to treat to harm (NNTH) for population risk of 1.9/1000 liveborn infants ${ }^{4}$

\section{Per 1000 liveborn infants (\%)}

Pooled odds ratio Risk of persistent pulmonary hypertension of the newborn Absolute risk difference NNTH (No of women)

SSRIs in late pregnancy:

\begin{tabular}{llll}
\hline 2.84 (trim and fill revised) & $5.40(0.54)$ & $3.50(0.35)$ & 286 \\
\hline 2.5 & $4.75(0.48)$ & $2.85(0.29)$ & 351 \\
\hline SSRIs in early pregnancy: & & & \\
\hline 1.23 & $2.34(0.23)$ & $0.44(0.04)$ & 2288 \\
\hline
\end{tabular}




\section{Figures}

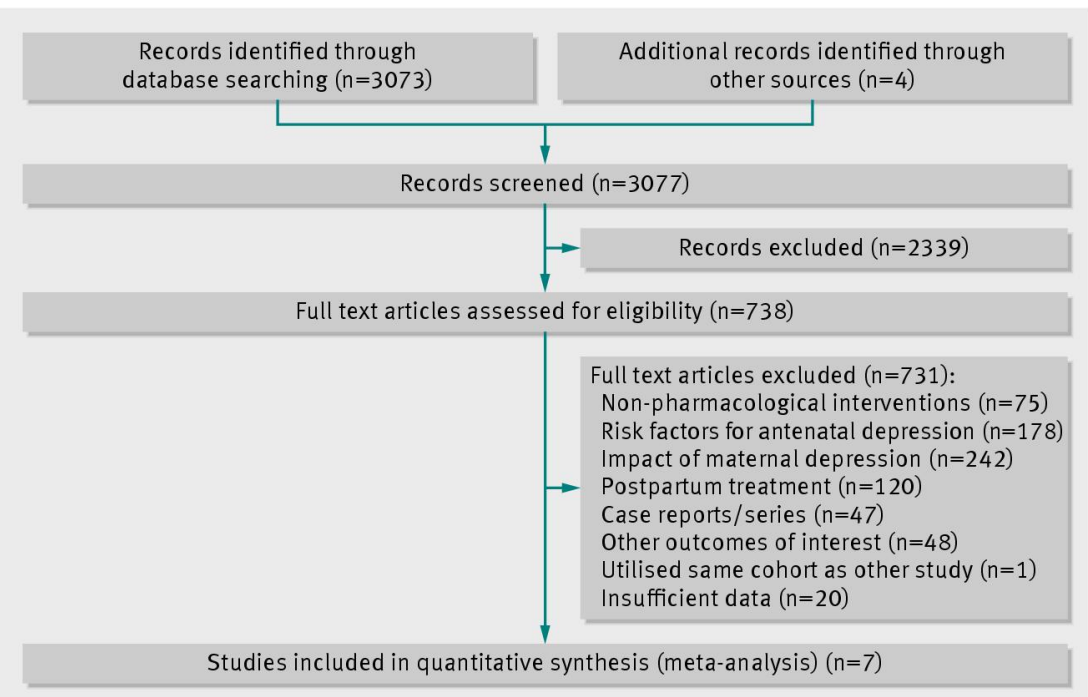

Fig 1 Identification of independent studies for inclusion in meta-analysis (adapted from PRISMA 2009 flow diagram ${ }^{30}$ )

Study

Chambers et al $2006^{2}$ Reis and Kallen $2010^{1}$ Kieler et al $2012^{3}$

Fixed effect model Random effects model Test for heterogeneity:

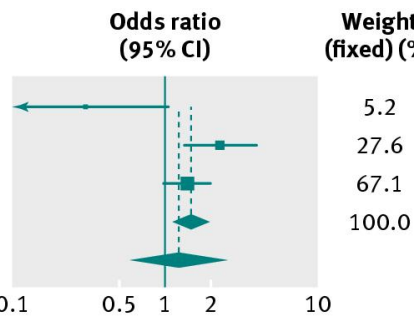

Weight

Odds ratio (random) $(\%) \quad(95 \% \mathrm{Cl})$

$\tau^{2}=0.31, P=0.011,\left.\right|^{2}=77.8 \%$

Fig 2 Exposure to selective serotonin reuptake inhibitors in early pregnancy and risk of persistent pulmonary hypertension of the newborn: meta-analysis of all studies

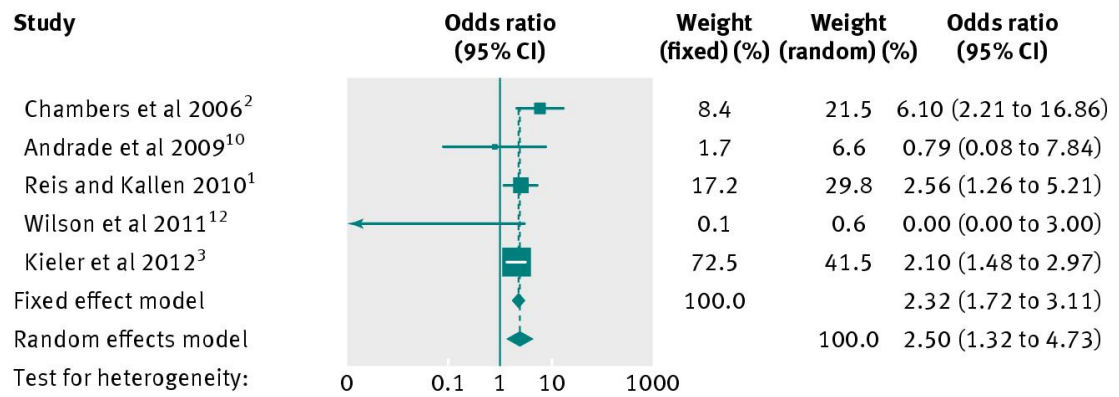

$\tau^{2}=0.22, P=0.081, I^{2}=51.9 \%$

Fig 3 Exposure to selective serotonin reuptake inhibitors in late pregnancy and risk of persistent pulmonary hypertension of the newborn: meta-analysis of all studies 